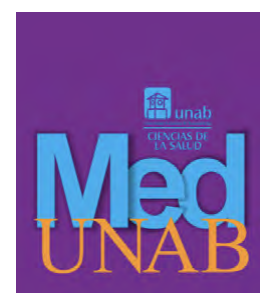

REVISTA DE LA FACULTAD

DE CIENCIAS DE LA SALUD

\title{
Caso clínico de enfermería: Implementación del modelo del logro de las metas a sujeto con diagnóstico de Sarcoma de Ewing
}

Clinical Case in Nursing: Implementing the Goal Attainment Model on Patients Diagnosed with Ewing Sarcoma

Caso clínico de enfermagem: Implementação de modelo de alcance de metas em um sujeito com diagnóstico de Sarcoma de Ewing

Andrés Leonardo Alvarado-Alvarado. Enf. ${ }^{1}$ iD, Ruby Alexandra HernándezMogollón. Enf. ${ }^{2}$ iD, Camila Andrea Bustamante-Gil. Enf. ${ }^{3}$ iD, Rosaida RodríguezHerrera. Enf., Esp. ${ }^{4}$ iD

1. Enfermero Universidad Autónoma de Bucaramanga. Bucaramanga, Santander, Colombia, Enfermero hospitalización adulto clínica FOSCAL Internacional. Bucaramanga. Santander, Colombia.

2. Enfermera Universidad Autónoma de Bucaramanga. Bucaramanga, Santander, Colombia Egresada, Diest. Bélgica.

3. Enfermera Universidad Autónoma de Bucaramanga. Bucaramanga, Santander, Colombia, Enfermera líder de la dimensión vida saludable y condiciones no transmisibles del plan de intervenciones PIC, área de promoción y prevención y enfermera asistencial área de urgencias. Hospital Local de Piedecuesta. Piedecuesta, Santander, Colombia

4. Enfermera Universidad Simón Bolívar, Especialista en atención de enfermería en cuidado crítico, Estudiante maestría en Ciencias de la enfermería, Universidad de Cuauhtémoc México, Enfermera UCI adultos clínica FOSCAL. Bucaramanga. Santander, Colombia, Docente cátedra Universidad Autónoma de Bucaramanga. Bucaramanga, Santander, Colombia.

Correspondencia. Andrés Leonardo Alvarado-Alvarado, Enfermero Universidad Autónoma de Bucaramanga, Santander, Colombia. Dirección. Calle 49 \# 3 - 31, Floridablanca, Santander, Colombia. Email. aalvarado510@unab. edu.co o alvaradoandres885@gmail.com

\section{INFORMACIÓN DEL ARTÍCULO:}

Artículo recibido: 11 de septiembre de 2019

Artículo aceptado: 01 de julio de 2020

DOI: https://doi.org/ 10.29375/01237047.3739

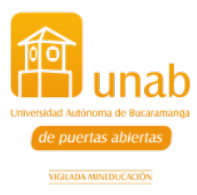

Cómo citar: Alvarado-Alvarado AL, Hernández-Mogollón RA, BustamanteGil CA, Rodríguez-Herrera R. Caso clínico de enfermería:Implementación del modelo del logro de las metas a sujeto con diagnóstico de Sarcoma de Ewing. MedUNAB. 2020;23(3): 471-482. doi: https://doi.org/10.29375/01237047.3739 


\title{
RESUMEN
}

Introducción. El sarcoma de Ewing se define como una neoplasia ósea de alta agresividad y su origen se atribuye a una patología de tipo endotelial vascular, caracterizándose como un tumor formado por células redondas indiferenciadas. Se ha descrito su presentación en lugares como el sistema nervioso central y paravertebral. Respectivamente afectando a niños y adolescentes. El objetivo de este trabajo es implementar el Proceso de Atención de Enfermería a sujeto de cuidado adolescente con diagnóstico médico de Sarcoma de Ewing. Metodología. Caso clínico de enfermería con un período de seguimiento de 3 meses a sujeto de cuidado con diagnóstico médico de Sarcoma de Ewing mediante la aplicación de la teoría del logro de las metas de Imogene King y la implementación del Proceso de Atención de Enfermería, correlacionando la taxonomía diagnóstica de la North American Nursing Diagnosis Association (NANDA), describiendo los resultados esperados de la Nursing Outcomes Classification (NOC) y la implementación de las intervenciones descritas en la Nursing Interventions Classification (NIC) respectivamente, en relación con los resultados esperados. Resultados. Se plantea el Proceso de Atención de Enfermería con diagnóstico de enfermería: Deterioro de la movilidad física; con el resultado de enfermería: Movimiento articular e intervenciones de enfermería: Terapia de ejercicios: Movilidad articular. Evidenciándose la mejoría de la fuerza muscular del sujeto de cuidado. Conclusión. Mediante las intervenciones realizadas se evidenció el progreso del sujeto de cuidado. La relación del Proceso de Atención de Enfermería, teoría de enfermería y las herramientas de diagnóstico, objetivo e intervenciones de enfermería permitieron el manejo holístico del sujeto de cuidado..

Palabras claves:

Sarcoma de Ewing; Proceso de enfermería; Diagnóstico de Enfermería; Planificación de Atención al sujeto de cuidado; Evaluación en Enfermería; Enfermería Pediátrica; Adolescente.

\begin{abstract}
Introduction. An Ewing Sarcoma is defined as a highly aggressive bone tumor. Its origin is attributed to a vascular endothelial pathology and it is characterized as a tumor made of undifferentiated round cells. Its presence has been described in places, such as the central nervous and paravertebral system. It equally affects children and adolescents. Objective. To implement Nursing Assistance Processes to adolescent patients with Ewing Sarcoma medical diagnoses. Methodology. A clinical case in nursing with a 3-month monitoring period on patients with Ewing sarcoma medical diagnoses. It is performed by applying Imogene King's Theory of Goal Attainment and implementing the Nursing Assistance Process, correlating the diagnostic taxonomy of the North American Nursing Diagnosis Association, describing the expected results of the Nursing Outcomes Classifications and implementing the interventions described in the Nursing Interventions Classification, respectively, when it comes to the expected results. Results. The Nursing Assistance Process is posed with a nursing diagnosis: Deterioration of physical mobility; with the nursing result: Joint movement and nursing interventions: Exercise therapy: Joint mobility. Improvement in the patient's muscular strength is evident. Conclusion. The patient's progress due to the performed interventions was evident. The Nursing Assistance Process, theory of nursing and diagnostic tools, as well as nursing goals and interventions, allowed comprehensively handling the patient.
\end{abstract}

Keywords:

Ewing Sarcoma; Nursing Process; Nursing Diagnosis; Planning Patient Assistance; Nursing Assessment; Pediatric Nursing; Adolescent.

\section{RESUMO}

Introdução. O sarcoma de Ewing é definido como uma neoplasia óssea altamente agressiva e sua origem é atribuída a uma patologia do tipo endotelial vascular, caracterizada como um tumor formado por células redondas indiferenciadas. Foi descrito seu aparecimento em locais como o sistema nervoso central e paravertebral. Afeta respectivamente crianças e adolescentes. Objetivo. Implementar um Processo de Assistência de Enfermagem a um sujeito do cuidado, adolescente, com diagnóstico médico de Sarcoma 
de Ewing. Métodos. Caso clínico de enfermagem com seguimento de 3 meses a um sujeito do cuidado com diagnóstico médico de Sarcoma de Ewing por meio da aplicação da teoria do alcance de metas de Imogene King e da implementação do Processo de Assistência de Enfermagem, correlacionando a taxonomia diagnóstica da North American Nursing Diagnosis Association (NANDA), descrevendo os resultados esperados da Nursing Outcomes Classification e a implementação das intervenções descritas na Nursing Intervention Classification respectivamente, em relação aos resultados esperados. Resultados. Propõe-se o Processo de Assistência de Enfermagem com diagnóstico de enfermagem: Deterioro da mobilidade física; com resultado de enfermagem: Movimento articular; e intervenções de enfermagem: Terapia por exercício: Mobilidade articular. Foi evidente a melhora na força muscular do sujeito do cuidado. Conclusão. Por meio das intervenções realizadas foi evidente a evolução do sujeito do cuidado. A relação do Processo de Assistência de Enfermagem, teoria de enfermagem e as ferramentas diagnósticas, objetivo e intervenções de enfermagem possibilitaram o manejo holístico do sujeito do cuidado.

Palavras-chave:

Sarcoma de Ewing; Processo de enfermagem; Diagnóstico de Enfermagem; Planificação de Assistência ao sujeito do cuidado; Avaliação em Enfermagem; Enfermagem Pediátrica; Adolescente.

\section{Introducción}

El sarcoma de Ewing fue definido en el año 1921 por James Ewing como una neoplasia de origen endotelial caracterizada por ser un tumor formado de células redondas e indiferenciadas $(1,2)$. Expresa su patogenia en la población pediátrica y adolescente en mayor porcentaje $(2,3)$. Es un tumor maligno de tejidos blandos poco frecuente y tiene su origen en las células mesenquimales (4). Su fisiopatología se desarrolla a nivel genético y se presenta como una translocación característica que, en la mayoría de los casos, implica los genes EWS de los cromosomas 22 y 11 con las expresiones $\mathrm{t}(11 ; 22)$ y (q24;q12), la cual resulta en la producción de proteínas quiméricas EWSR1-FLI1 $(3,4)$. Los sujetos de cuidado con este sarcoma presentan una clínica como: dolor, fiebre, leucocitosis, signos focales erróneos de un proceso infeccioso, masa tumoral suave, fracturas patológicas y síntomas neurológicos $(5-6,14)$.

\section{Epidemiología}

El sarcoma de Ewing es uno de los tumores sólidos más comunes en la población pediátrica (7), encontrándose más comúnmente en la población masculina, en proporción 2:1 con respecto a la femenina. En la población adolescente de los Estados Unidos, este tipo de sarcoma debuta mayormente en huesos, aunque puede aparecer en otras zonas anatómicas como la pelvis $(26.6 \%)$, las extremidades inferiores $(23.1 \%)$ o el tórax $(20.3 \%)$, con metástasis pulmonar del 70.6\% en este grupo de edad $(8,9)$. Los sarcomas de Ewing de cabeza y cuello no son muy comunes; se reportan del $3 \%$ al $9 \%$ de casos. En un estudio desarrollado en Estados
Unidos con 183 pacientes entre los años 1993 a 2013 se encontró que el tejido óseo más afectado son los huesos del cráneo, de la cara, las articulaciones asociadas $(40 \%)$ y el tejido conectivo subcutáneo junto con otros tejidos blandos (30\%). La media de su diagnóstico es de 23.4 años, es prevalente en población blanca $(67.6 \%)$ e hispana $(21.4 \%)$ y muy raro en la raza asiática $(6 \%)$ o negra $(3.9 \%)$. Además, tiene una extensión local del tumor primario del $65.5 \%(10-13)$.

La teoría de Imogene King (Figura 1) define enfermería como un proceso de acción, reacción e interacción; fortaleciendo las relaciones entre el profesional de enfermería con el sujeto de cuidado, y así mismo ofreciendo información acerca de las percepciones mutuas por parte de la enfermera y el sujeto $(15,16)$. El Proceso de Atención de Enfermería en el cuidado de una persona con sarcoma de Ewing u otro tipo de cáncer permite obtener datos acerca de la persona, su entorno y su capacidad social, al igual que identificar problemas del individuo, su familia y su comunidad. Este proceso se relaciona con la taxonomía de la Enfermería, que se ve marcada por uno o más diagnósticos, así como un objetivo propuesto a corto, mediano o largo plazo e intervenciones encaminadas a necesidades específicas, basadas en conocimientos científicos. Así mismo, la labor de enfermería permite abordar la teoría de Imogene King: el logro de metas mediante el fortalecimiento de redes de apoyo en el marco de las buenas relaciones familiares como eje para la recuperación de la salud del sujeto de cuidado. El objetivo de este estudio es implementar el Proceso de Atención de Enfermería (17), a sujeto de cuidado adolescente con diagnóstico de sarcoma de Ewing, mediante la aplicación de la teoría de Imogene King. 
Figura 1. Esquema de la teoría de enfermería del logro de las metas de Imogene King

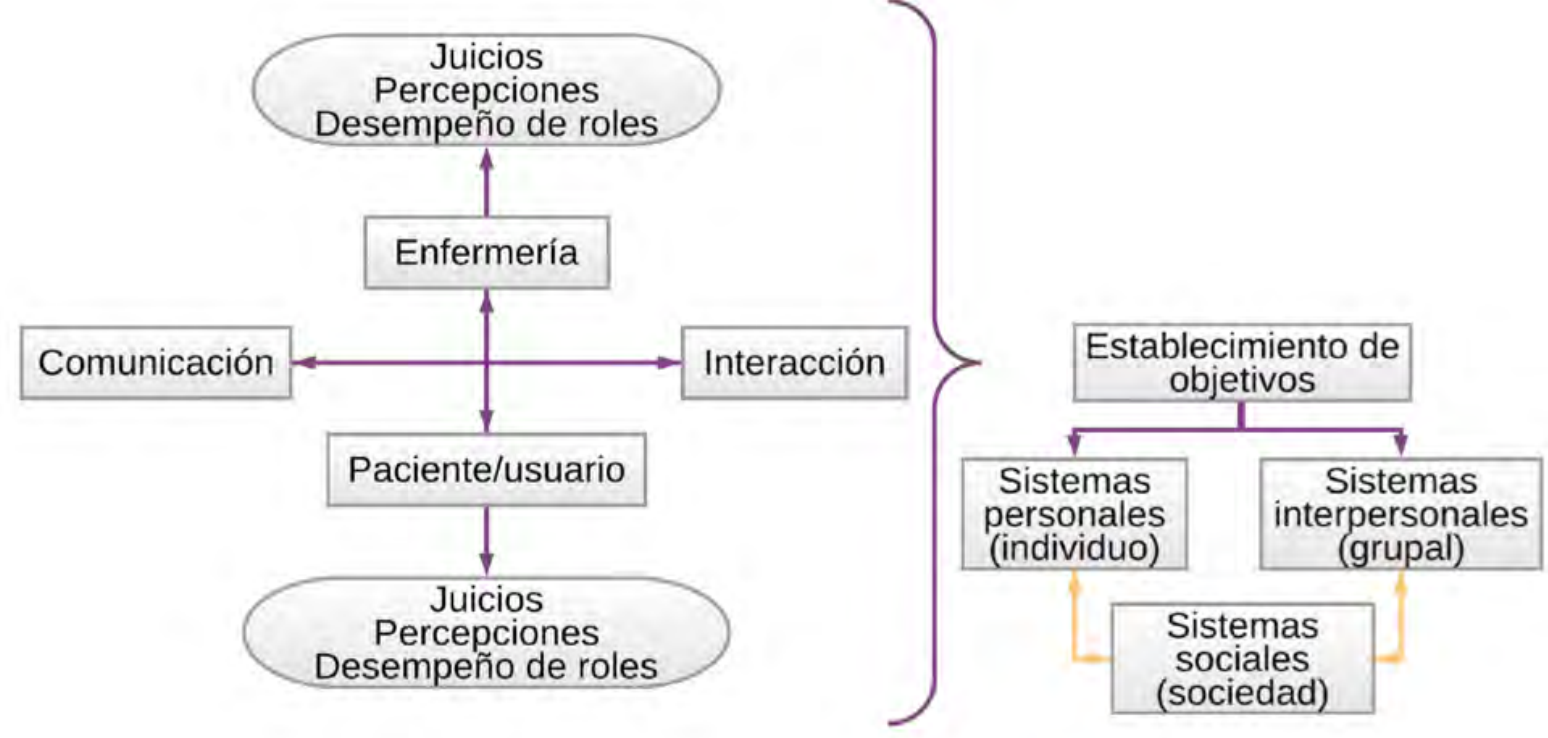

Fuente: elaboración propia de los autores.

\section{Metodología}

Presentación de caso clínico de enfermería con período de seguimiento de 3 meses a un sujeto de cuidado adolescente con diagnóstico médico de sarcoma de Ewing mediante la aplicación de la teoría del logro de las metas de Imogene King y la implementación del Proceso de Atención de enfermería.

\section{Consideraciones éticas}

Considerando los aspectos éticos establecidos por el Tribunal Nacional Ético de Enfermería de Colombia en la ley 911 de 2004, que dicta la responsabilidad deontológica de la profesión de Enfermería, de acuerdo a los artículos 4, 29 y 30, cuyo fundamento es salvaguardar la dignidad, la integridad y los derechos de los seres humanos como principio ético fundamental, se informa y se solicita el consentimiento informado a la persona y la familia, obteniendo el consentimiento de los padres y el asentimiento del menor.

\section{Presentación del caso clínico}

Adolescente de 13 años de edad que consulta por 2 meses de evolución de dolor en región cervical que compromete cuello, hombro izquierdo y la movilidad del mismo, limitando la fuerza de las cuatro extremidades.
Es hospitalizado el 6 de marzo de 2018, se le realiza resonancia magnética valorada por neurocirugía el 8 de marzo dando como resultado una masa a nivel del canal epidural desde $\mathrm{C} 2$ hasta C7. Posteriormente, se le realiza cirugía de emergencia para descompresión y resección de masa tumoral desde C2 a C7. Después es remitido a la Unidad de Cuidados Intensivos (UCI) para recuperación. El 11 de marzo es trasladado de la UCI al área de hospitalización y el 16 de marzo el área de patología confirma el diagnóstico médico de sarcoma de Ewing. El 19 de marzo se inicia manejo con quimioterapia en el servicio de Hemato-oncología pediátrica hasta el 18 de mayo.

\section{Resultados}

La valoración e identificación del diagnóstico de enfermería se realizó mediante la implementación de la teoría del logro de las metas de Imogene King y el Proceso de Atención de Enfermería (PAE), incluyendo el dominio NANDA más alterado.

Etapa 1: Valoración del sujeto de cuidado mediante los sistemas de la teoría de Imogene King (18). Esta etapa se implementó con base a los tres sistemas y subsistemas que propone Imogene King en su teoría del logro de las metas (tabla 1). 
Tabla 1. Valoración del sujeto de cuidado mediante los sistemas de la teoría de Imogene King.

\section{SISTEMA PERSONAL \\ SISTEMA INTERPERSONAL}

La percepción: Adolescente de 13 años, hermano mayor, que refiere poca actividad

física y pocas relaciones de amistad, educación formal interrumpida.
La interacción: Sujeto de cuidado colaborador durante la anamnesis y valoración.

\section{SISTEMA SOCIAL}

La organización:

Núcleo familiar: Madre, padrastro y hermano menor.
La imagen corporal: Constitución corporal delgada, de conformación simétrica, con edad aparente a su edad cronológica, estatura de 1.60 metros, lenguaje adecuado a su nivel sociocultural.

Alteración corporal derivada de pérdida de movimiento de las extremidades, tono muscular y fuerza, que le impiden realizar actividades de la vida diaria. Valoración de la fuerza de las extremidades se desarrolló mediante la escala Daniels (15) (Tablas 2 y 3 )

El tiempo: Refiere que su plan a futuro es trabajar y no estudiar.
La comunicación: Relación frecuente con abuelos, la interacción familiar provee un estímulo positivo para el proceso de valoración.
Ubicación diaria: En casa de los abuelos, convive con su tía, primos y bisabuela.

Alteración de la interacción social a nivel comunitario: escolar, comunitario y de ocio debido al proceso patológico.

El estrés: Refiere que la hospitalización es una situación difícil que enfrenta con paciencia y apoyo materno. Considera tener un poco de ansiedad y miedo por su diagnóstico oncológico.

El aprendizaje: Sin problemas para el aprendizaje de cosas nuevas, pero sí para concentrarse: dificultad para mantener la atención por tiempo prolongado
La autoridad y el poder: $\mathrm{Al}$ ser menor de edad, la madre es quien posee la potestad, ella toma decisiones con asentimiento del sujeto de cuidado.

El yo: Se describe a sí mismo como un joven responsable y amable, se acepta tal como es.

Fuente: elaboración propia de los autores.

\section{Dominios NANDA alterados:}

Dentro de la valoración por dominios aplicada al sujeto de cuidado hubo 4 dominios alterados, el dominio de Nutrición reflejado en la alta ingesta de carbohidratos, azúcares y grasas que refiere su cuidador y él mismo, al examen físico se presenta con índice de masa corporal de $26 \mathrm{Kg} / \mathrm{m} 2$ clasificado en sobrepeso; el dominio de Eliminación e intercambio que al examen físico presenta diuresis asistida por cateterismo vesical cada 6 horas por 3 semanas; el dominio de Afrontamiento $\mathrm{y}$ tolerancia al estrés en que el sujeto de cuidado se encuentra (temeroso a su diagnóstico médico, junto con la estancia hospitalaria, y además refiere que es una situación difícil); y el dominio de Actividad y reposo que, mediante el proceso de atención de enfermería en sus etapas 1 y 2 , se priorizó mediante las características definitorias y relacionados. (Tabla 3). Al igual es pertinente relacionar este dominio, que a continuación se describirá a profundidad, con los hallazgos relevantes en el sujeto de cuidado, que es importante intervenir para que el adolescente pueda continuar su vida cotidiana normalmente. 
Tabla 3. Diagnóstico de Enfermería NANDA, indicadores de resultados iniciales y esperados NOC y plan de cuidados NIC.

\section{Sección A}

\section{Diagnóstico de Enfermería}

\section{Dominio 4:}

Actividad /

Reposo.

\section{Clase 2:}

Actividad/ Ejercicio.

\section{Etiqueta diagnóstica}

Deterioro de la movilidad física (00085)

\section{Características definitorias:}

Dificultad para girarse.

Limitación de la amplitud de movimientos.

Dificultad para la marcha

Relacionados:

Disminución de la fuerza muscular. Disminución de la resistencia.

Definición: Limitación del movimiento físico independiente intencionado del cuerpo o de una o más extremidades.

\section{Sección B}

Etiqueta diagnóstica: Deterioro de la movilidad física (00085).

Dominio 4: Actividad/ Reposo. Clase 2: Actividad/ Ejercicio.

Resultado NOC: Movimiento articular: hombro (0219).

Objetivo de enfermería: El sujeto de cuidado mejorará el movimiento articular a través de los indicadores: flexión hacia delante de $180^{\circ}$, extensión de $50^{\circ}$, rotación externa de $90^{\circ}$, rotación interna de $90^{\circ}$ y abducción de $180^{\circ}$ en un lapso de tiempo de 3 meses.

Escala Likert: Desviación grave del rango normal hasta sin desviación del rango normal

\begin{tabular}{|c|c|c|c|c|}
\hline $\begin{array}{l}\text { Indicadores: } \\
\text { Aplicados al } \\
\text { miembro superior } \\
\text { izquierdo. }\end{array}$ & $\begin{array}{l}\text { 1: Contracción } \\
\text { muscular, pero sin } \\
\text { movimiento. }\end{array}$ & $\begin{array}{l}\text { 2: Gama total de } \\
\text { movimientos, } \\
\text { pero no contra } \\
\text { gravedad. }\end{array}$ & $\begin{array}{l}\text { 3: Gama total de } \\
\text { movimientos contra } \\
\text { la gravedad, pero sin } \\
\text { resistencia. }\end{array}$ & $\begin{array}{l}\text { 4: Gama total de } 5: \text { Es normal, gama } \\
\text { movimientos contra la total de movimientos } \\
\text { gravedad, pero débil. } \quad \text { contra la gravedad. }\end{array}$ \\
\hline $\begin{array}{l}\text { Flexión hacia } \\
\text { delante de } 180^{\circ}\end{array}$ & & I & & E \\
\hline Extensión de $50^{\circ}$ & & I & & E \\
\hline $\begin{array}{l}\text { Rotación externa } \\
\text { de } 90^{\circ}\end{array}$ & & I & & E \\
\hline $\begin{array}{l}\text { Rotación interna } \\
\text { de } 90^{\circ}\end{array}$ & & I & & E \\
\hline $\begin{array}{l}\text { Abducción } \\
\text { de } 180^{\circ}\end{array}$ & & I & & $\mathrm{E}$ \\
\hline
\end{tabular}

NOC Inicial: $40 \%$

NOC Esperado: $80 \%$

$\begin{array}{cccccc}\begin{array}{c}\text { Indicadores: } \\ \text { Aplicados al }\end{array} & \begin{array}{c}\text { 1: Contracción } \\ \text { muscular, pero sin } \\ \text { miembro superior } \\ \text { movimiento }\end{array} & \begin{array}{c}\text { total de } \\ \text { movimientos, } \\ \text { pero no contra } \\ \text { gravedad. }\end{array} & \begin{array}{c}\text { 3: Gama total de } \\ \text { movimientos contra } \\ \text { la gravedad pero sin } \\ \text { resistencia }\end{array} & \begin{array}{c}\text { 4: Gama total de } \\ \text { movimientos contra } \\ \text { la gravedad, pero } \\ \text { débil. }\end{array} & \begin{array}{c}\text { 5: Es normal, } \\ \text { gama total de } \\ \text { movimientos } \\ \text { contra la gravedad. }\end{array}\end{array}$

Flexión hacia

delante de $180^{\circ}$
I

I
E

E 


\begin{tabular}{|c|c|c|}
\hline $\begin{array}{l}\text { Rotación externa } \\
\text { de } 90^{\circ}\end{array}$ & I & $\mathrm{E}$ \\
\hline $\begin{array}{l}\text { Rotación interna } \\
\text { de } 90^{\circ}\end{array}$ & I & $\mathrm{E}$ \\
\hline $\begin{array}{c}\text { Abducción de } \\
180^{\circ}\end{array}$ & I & $\mathrm{E}$ \\
\hline
\end{tabular}

NOC Inicial: $60 \%$

NOC Esperado: $100 \%$

Intervención NIC: Terapia de ejercicios: movilidad articular

Actividades:

- Ayudar al paciente a colocarse en una posición óptima para el movimiento articular pasivo/activo.

- Fomentar la realización de ejercicios de rango de movimiento de acuerdo con un programa regular, planificado.

- Realizar ejercicios pasivos o asistidos de rango de movimiento.

- Ayudar al paciente a desarrollar un programa de ejercicios activos de rango de movimiento.

- Enseñar al paciente/familia a realizar de forma sistemática los ejercicios de rango de movimiento pasivos o activos.

$\mathrm{I}=$ NOC Inicial

$\mathrm{E}=$ NOC Esperado.

NANDA: North American Nursing Diagnosis Association

NOC: Nursing Outcomes Classification

NIC: Nursing Interventions Classification

Fuente: elaboración propia de los autores, con base en datos obtenidos de la NNNCONSULT (21).

Actividad/Reposo: En la valoración física del sujeto de cuidado se evaluó la pérdida de movimiento de las extremidades, tono muscular y fuerza, lo que le dificulta realizar actividades de la vida diaria como tomar un objeto y elevarlo hasta el nivel de la cabeza, apoyarse en alguna superficie de forma firme, o incluso asearse por sí mismo, la valoración de la fuerza de las extremidades se desarrolló mediante la escala Daniels (19), que se describe en la Tabla 2 (Sección A). En la sección $\mathrm{B}$ de dicha tabla se muestran los resultados obtenidos respectivamente a la valoración de la fuerza muscular.

Etapa 2: Diagnóstico de enfermería.

Con base en los resultados de la valoración por dominios se correlacionó un diagnóstico de enfermería evidenciado en tres características definitorias y dos relacionados de la NANDA $(20,21)$ que se describe en la sección A de la Tabla 3.

Etapa 3: Planteamiento del cuidado.

Para el diagnóstico identificado se desarrolló el objetivo NOC (22) con los indicadores asociados y la intervención
NIC con sus actividades a realizar (23). Los indicadores NOC se plantearon mediante la integración de la escala Daniels a la escala Likert, describiendo la puntuación de los resultados dependiendo de la fuerza muscular de las extremidades superiores del sujeto de cuidado. (Tabla 3 sección B). El objetivo de enfermería planteado es: El sujeto de cuidado mejorará el movimiento articular a través de los indicadores: flexión hacia delante de $180^{\circ}$, extensión de $50^{\circ}$, rotación externa de $90^{\circ}$, rotación interna de $90^{\circ}$ y abducción de $180^{\circ}$ en un lapso de tiempo de 3 meses.

Las actividades e intervenciones planteadas se encaminaron al NOC movimiento articular: hombro. Estos indicadores se correlacionaron con la puntuación de la escala Likert de 1 a 5 , respectivamente, con los grados de la escala Daniels, iniciando desde el grado 1: "contracción muscular, pero sin movimiento" hasta el grado 5: "es normal, gama total de movimientos contra la gravedad". Esto se valoró con cada indicador NOC de movimiento articular de hombro implementado a los movimientos fisiológicos normales como son: flexión, extensión, rotación y abducción. Los resultados iniciales obtenidos comprobaron que la extremidad superior izquierda es la más comprometida conforme a la 
pérdida de la fuerza muscular. Los puntajes esperados a cumplir en el sujeto de cuidado se plantearon conforme a mejorar dos grados la fuerza muscular.

Tabla 2. Ítems clasificatorios escala Daniels y resultados valoración de la fuerza muscular Daniels.

\section{Sección A}

\section{Escala de fuerza muscular}

Es normal, gama total de movimientos contra la gravedad.

Grado 5

Gama total de movimientos contra la gravedad, pero débil.

Grado 4

Gama total de movimientos contra la gravedad, pero sin resistencia.

Grado 3

Gama total de movimientos, pero no contra gravedad.

Grado 2

Contracción muscular, pero sin movimiento.

Grado 1

Ausencia de contractibilidad.

Grado 0

Sección B

\begin{tabular}{|cc|}
\hline \multicolumn{2}{|c|}{ Resultados } \\
\hline Extremidad & Grado obtenido \\
\hline Miembro superior derecho & Grado 3 \\
\hline Miembro superior izquierdo & Grado 2 \\
\hline Miembro inferior derecho & Grado 4 \\
\hline Miembro inferior izquierdo & Grado 4 \\
\hline
\end{tabular}

Fuente: escala obtenida del artículo original de Capelari et al. (29).

\section{Evidencia literaria}

- Ayudar al sujeto de cuidado a colocarse en una posición óptima para el movimiento: Una postura correcta es toda aquella que presenta una adecuada alineación de las estructuras corporales, conservando el trayecto normal de la línea de gravedad con un máximo de eficiencia fisiológica y biomecánica, requiriendo un mínimo esfuerzo y gasto energético (24).
- Fomentar la realización de ejercicios de rango de movimiento de acuerdo con un programa regular, planificado: La actividad física disminuye la influencia desfavorable de la actividad motora disminuida, previenen las complicaciones, intensifican las reacciones de defensa del organismo durante la enfermedad y contribuyen al desarrollo de los mecanismos compensadores. Además, influyen favorablemente en la psiquis del paciente, fortalecen sus cualidades volitivas y la esfera emocional (25).

- Realizar ejercicios pasivos o asistidos de rango de movimiento: El ejercicio con movimiento pasivo es el que realiza el examinador sin la ayuda de la acción muscular activa de la persona examinada, que puede o no estar consciente. No existe contracción muscular voluntaria, por lo que se requiere una fuerza externa para ejecutarlo (26).

- Ayudar al sujeto de cuidado a desarrollar un programa de ejercicios activos de rango de movimiento: Dentro de las secuelas de los tumores podemos encontrar la pérdida de la fuerza muscular. Esta fuerza puede ser recuperada por medio de la ayuda de terapia de ejercicios activos que con adherencia permiten mejorar el rango de movimiento de las extremidades (27).

- Enseñar al sujeto de cuidado/familia a realizar de forma sistemática los ejercicios de rango de movimiento pasivos o activos: El entrenamiento funcional es un método para volver a entrenar el sistema motor mediante la práctica repetitiva de tareas funcionales, en un intento de restablecer la capacidad del usuario de realizar actividades de la vida diaria y participar en actividades específicas (28).

Etapa 4: Ejecución del plan.

La ejecución del plan de enfermería se implementó mediante la aplicación de los sistemas de la teoría de Imogene King, correlacionando el sistema social conformado por la familia y el poder que le genera al sistema personal que se desarrolla en el sujeto de cuidado. La familia como sistema (en la teoría) aporta gran avance en la atención al sujeto de cuidado, dado que es su principal recurso socioafectivo, al mismo tiempo la relación de los sistemas enfoca a que el individuo pueda mejorar su capacidad física de manera estructural. 
Etapa 5: Evaluación.

Los resultados obtenidos en la implementación del Proceso de Atención de Enfermería en un seguimiento a las actividades por un período de 3 meses, con relación a cada objetivo NOC, se evidencian en la Tabla 4. Consecutivamente en la evaluación de las 4 etapas anteriores, desde el enfoque en la valoración mediante dominios NANDA y la teoría de enfermería se priorizó un problema desde la función fisiológica y el desarrollo teórico de los sistemas sociales y personales del logro de metas.

Los resultados obtenidos conforme al NOC inicial, final y esperado se describen en la Figura 2, enfatizando que para el miembro superior izquierdo se logró el $80 \%$ y para el miembro superior derecho el 100\% cumpliendo con los puntajes esperados y la mejoría del sujeto de cuidado.

Tabla 4. Plan de cuidados, indicadores de resultados finales NOC.

Etiqueta diagnóstica: Deterioro de la movilidad física (00085)

Dominio 4: Actividad/ Reposo. Clase 2: Actividad/ Ejercicio

Resultado NOC: Movimiento articular: hombro (0219)

Objetivo de enfermería: El sujeto de cuidado mejorará el movimiento articular a través de los indicadores: flexión hacia delante de $180^{\circ}$, extensión de $50^{\circ}$, rotación externa de $90^{\circ}$, rotación interna de $90^{\circ}$ y abducción de $180^{\circ}$ en un lapso de tiempo de 3 meses.

Escala Likert: Desviación grave del rango normal hasta sin desviación del rango normal

$\begin{array}{cccccc}\text { Indicadores: Aplicados } & \text { 1: Contracción } \\ \text { al miembro superior } & \begin{array}{c}\text { muscular, pero } \\ \text { izquierdo. }\end{array} & \begin{array}{c}\text { movimientos, } \\ \text { mero no contra }\end{array} & \begin{array}{c}\text { 3: Gama total } \\ \text { de movimientos } \\ \text { contra la gravedad }\end{array} & \begin{array}{c}\text { 4: Gama total movimientos } \\ \text { contra la }\end{array} & \begin{array}{c}\text { 5: Es normal, } \\ \text { gama total de } \\ \text { movimientos }\end{array} \\ \begin{array}{c}\text { gravedad. } \\ \text { pero sin resistencia }\end{array} & \begin{array}{c}\text { gravedad, pero } \\ \text { débil. }\end{array} & \begin{array}{c}\text { contra la } \\ \text { gravedad. }\end{array}\end{array}$

Flexión hacia delante de $180^{\circ}$

\section{F}

$\mathrm{F}$

F

$\mathrm{F}$

F

Abducción de $180^{\circ}$

NOC Inicial: $40 \%$

NOC Esperado: $80 \%$

NOC Final: $\mathbf{8 0 \%}$

\begin{tabular}{|c|c|c|c|c|c|}
\hline $\begin{array}{l}\text { Indicadores: } \\
\text { Aplicados al miembro } \\
\text { superior derecho. }\end{array}$ & $\begin{array}{l}\text { 1: Contracción } \\
\text { muscular, pero } \\
\text { sin movimiento. }\end{array}$ & $\begin{array}{l}\text { 2: Gama total de } \\
\text { movimientos, } \\
\text { pero no contra } \\
\text { gravedad. }\end{array}$ & $\begin{array}{c}\text { 3: Gama total } \\
\text { de movimientos } \\
\text { contra la gravedad } \\
\text { pero sin resistencia }\end{array}$ & $\begin{array}{c}\text { 4: Gama total } \\
\text { de movimientos } \\
\text { contra la } \\
\text { gravedad, pero } \\
\text { débil. }\end{array}$ & $\begin{array}{l}\text { 5: Es normal, } \\
\text { gama total de } \\
\text { movimientos } \\
\text { contra la } \\
\text { gravedad. }\end{array}$ \\
\hline Flexión hacia delante de $180^{\circ}$ & & & & & $\mathrm{F}$ \\
\hline Extensión de $50^{\circ}$ & & & & & $\mathrm{F}$ \\
\hline Rotación externa de $90^{\circ}$ & & & & & $\mathrm{F}$ \\
\hline Rotación interna de 90 & & & & & $\mathrm{~F}$ \\
\hline \multicolumn{6}{|l|}{ Abducción de $180^{\circ}$} \\
\hline \multicolumn{6}{|l|}{ NOC Inicial: $60 \%$} \\
\hline \multicolumn{6}{|l|}{ NOC Esperado: $100 \%$} \\
\hline \multicolumn{6}{|l|}{ NOC Final: 100\% } \\
\hline $\mathrm{F}=$ NOC Final & & & & & \\
\hline
\end{tabular}

Fuente: elaboración propia de los autores, con base en datos obtenidos de la NNNCONSULT (21). 
Figura 2. Resultados del Proceso de Atención de Enfermería

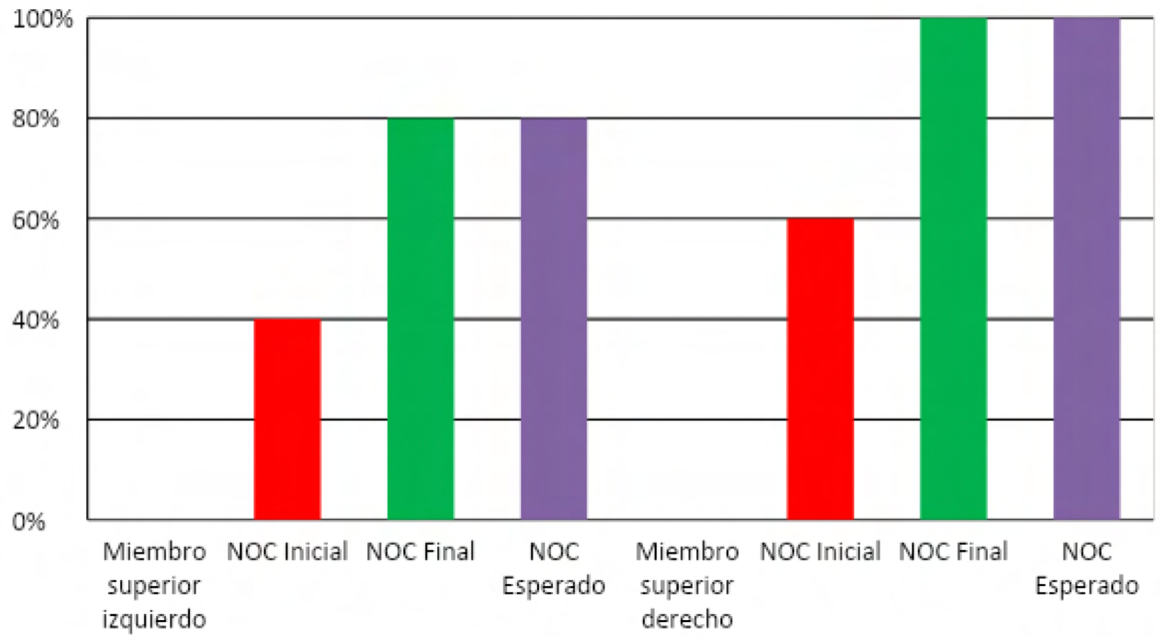

NANDA: North American Nursing Diagnosis Association

NOC: Nursing Outcomes Classification

NIC: Nursing Interventions Classification.

Fuente: elaboración propia de los autores.

\section{Conclusión}

El Proceso de Atención de Enfermería permite el cuidado del paciente, su familia y su comunidad en todos sus aspectos e interacciones entre sí. Esto, unido a la integración de las teorías de enfermería, que aportan la base teórica al cuidado, mejora la calidad y el enfoque del cuidado en patologías o situaciones de distintos ciclos vitales específicos.

Teniendo en cuenta que el diagnóstico médico genera un gran impacto en todos los ámbitos y áreas del sujeto de cuidado, familia y comunidad, y que muchas veces se genera un desbalance en las diferentes áreas de la vida, hace que la intervención del profesional de enfermería se vuelva fundamental para el apoyo en el proceso de la enfermedad.

La patología oncológica generó una restricción de movilidad, haciendo que se presentara un cuadro de ansiedad y temor ante la situación de debilidad e incapacidad de realizar actividades que no representaban ninguna dificultad en el sujeto de cuidado y su familia. Por ende, la intervención del profesional de enfermería constituyó un alivio en las cargas de los diferentes miembros de la familia, resolución de dudas, temores respecto a la patología, tratamiento, cuidados especiales, la implicación física y psicológica tanto del sujeto de cuidado como de la familia en la rehabilitación. Esto dio como resultado la mejoría de la movilidad en el paciente, que aunado al tratamiento oncológico y a una mejora del autocuidado, resultó en el manejo de las herramientas necesarias para el desarrollo de sus actividades y cuidados sin la implicación directa de enfermería en el cuidado.

Se recomienda a los profesionales de enfermería de las diferentes áreas de la salud incluir el Proceso de Atención de Enfermería en su quehacer diario e implementar las distintas teorías y modelos de enfermería para desarrollar su plan de cuidado, debido a que en la implementación de la taxonomía de enfermería se puede diagnosticar a un sujeto de cuidado (con base en una etiqueta diagnóstica), y desarrollar un plan de acción mediante actividades que generen resultados objetivos (que pueden ser o no satisfactorios). En el caso de obtener resultados no satisfactorios, se pueden plantear objetivos a largo plazo o incluso desarrollar mejores intervenciones y actividades con mejores grados de recomendación y niveles de evidencia, para mejorar el estado de salud de los sujetos de cuidado.

\section{Financiamiento:}

Ninguno.

\section{Conflictos de interés:}

Ninguno. 


\section{Agradecimientos:}

Los autores agradecen la participación de los enfermeros Daniel Murillo, Angie Rosas y Ginary Orduz, quienes apoyaron en la aplicación del Proceso de Atención de Enfermería.

\section{Referencias}

1. Tuan Sharif SE, Alzallal O, Wan Ismail WF, Md Zin RR. Ewing Sarcoma presenting as a gluteal mass in adult woman: Diagnostic approach and its challenges. Malaysian J Pathol. 2020; 42(1): 121-125. [citado May 2020]. URL: http://www.mjpath.org.my/2020/ v42n1/Ewing-sarcoma.pdf

2. Polimera H, Moku P, Piedra Abusharar S, Vasekar M, Chintanaboina J. Metastasis of Ewing Sarcoma to the Pancreas: Case Report and Literature Review. Case Rep Oncol Med. 2020. [citado May 2020]. Disponible en: https://doi.org/10.1155/2020/7075048

3. Pasha HA, Ghaloo SK, Wasif M, Siddiqui MI, Din NU. Ewing Sarcoma of Larynx: A Rare Case in a 5-Year-Old Boy. Turk Arch Otorhinolaryngol. 2020; 58(1): 65-68. [citado May 2020]. Disponible en: https://doi.org/10.5152/tao.2020.4839

4. Ventura N, Cebreros D, Miragall L, Ruiz J, Puglia V. Sarcoma de Ewing extraóseo en cabeza y cuello: revisión de la literatura a propósito de un caso. Rev esp cir oral maxilofac. 2017; 39(2): 105-112. [citado Sep 2019]. Disponible en: https://doi.org/10.1016/j. maxilo.2016.03.003

5. Montoya $\mathrm{C}$, Rodríguez J, Fernández MJ, Troncoso D, Cañas A, Moreno O, Henríquez B, Rojas A. Epigenetic control of the EWS-FLI1 promoter in Ewing's sarcoma. Oncology reports 2020; 43: 11991207. [citado May 2020]. Disponible en: https://doi. org/10.3892/or.2020.7489

6. Miller HE, Gorthi A, Bassani N, Lawrence LA, Iskra BS, Bishop AJ. Reconstruction of Ewing Sarcoma Developmental Context from Mass-Scale Transcriptomics Reveals Characteristics of EWSR1FLI1 Permissibility. Cancers. 2020; 12, 948. [citado May 2020]. Disponible en: https://doi.org/10.3390/ cancers 12040948

7. Salguero Aranda C, Amaral AT, Olmedo Pelayo J, Diaz Martín J, Álava E. Breakthrough Technologies Reshape the Ewing Sarcoma Molecular Landscape. Cells. 2020; 9, 804. [citado May 2020]. Disponible en: https://doi.org/10.3390/cells9040804

8. Rios L, Vásquez L, Silva J, Sialer L, Maza I, Oscanoa $\mathrm{M}$, et al. Factores pronósticos y sobrevida en pacientes menores de 18 años con tumores de la familia del sarcoma de Ewing: experiencia de 10 años. Horiz Med. 2017; 17(4): 6-14. [citado Sep 2019]. Disponible en: https://doi.org/10.24265/horizmed.2017.v17n4.02
9. Davis RD, Berg E, Ranjit E, Bhandari P, Sapra A. Clinicians Beware, Ewing's Sarcoma After 60 Is Elusive and Rare!. Cureus. 2020; 12(1). [citado May 2020]. Disponible en: https://doi.org/10.7759/ cureus.6768

10. Ellis M, Gerry D, Neskey D, Lentsch E. Ewing Sarcoma of the Head and Neck: Analysis of 183 Cases With Comparison to Non-Head and Neck Sites. Ann Otol Rhinol Laryngol. 2017; 126(3): 179184. [Citado Ene 2020]. Disponible en: https://doi. org $/ 10.1177 / 0003489416681322$

11. Shi J, Yang J, Ma X, Wang X. Risk factors for metastasis and poor prognosis of Ewing sarcoma: a population based study. J Orthop Surg Res. 2020; 15(1): 88. [citado May 2020]. Disponible en: https:// doi.org/10.1186/s13018-020-01607-8

12. Takami Y, Aga F, Mitamura K, Norikane T, Okuda H, Yamamoto Y, Miyake M, Nishiyama Y. A Case of Ewing Sarcoma of the Mandible on 18F-FDG PET/ CT. Asia Ocean J Nucl Med Biol. 2020; 8(1): 8487. [citado May 2020]. Disponible en: https://doi. org/10.22038/aojnmb.2019.13876

13. Soni K, Elhence P, Kesarwani A, Rajan N. Primary Ewing's Sarcoma of Zygoma. Ann Maxillofac Surg. 2019; 9(2): 419-422. [citado May 2020]. URL: $\quad$ https://www.ncbi.nlm.nih.gov/pmc/articles/ PMC6933977/?report=printable

14. Keskin T, Bakaric A, Waszyk P, Boulay G, Torsello $\mathrm{M}$, Cornaz Buros $\mathrm{S}$, et al. LIN28B Underlies the Pathogenesis of a Subclass of Ewing Sarcoma. Cell Reports. 2020; 31(2): 4567-4583. [citado May 2020]. Disponible en: https://doi.org/10.1016/j. celrep.2019.12.053

15. Adib-Hajbaghery M, Tahmouresi M. Nurse-patient relationship based on the Imogene king's theory of goal attainment. Nurs Midwifery Stud. 2018; 7 :1414. [citado May 2020]. Disponible en: https://doi. org/10.4103/nms.nms $10 \quad 17$

16. McQueen L, Cockroft M, Mullins N. Imogene King's Theory of Goal Attainment and the Millennial Nurse: An Important Mentoring Tool for Nurse Educators. Teaching and Learning in Nursing. 2017; 12(3) 223225. [citado May 2020]. Disponible en: http://dx.doi. org/10.1016/j.teln.2017.03.003

17. Silva Fhon J, Ramón Cordova S, Vergaray Villanueva S, Palacios Fhon V, Partenazi Rodrigues R. Percepción del paciente hospitalizado respecto a la atención de enfermería en un hospital público. Enfermería Universitaria. 2015. [citado May 2020]. Disponible en: http://dx.doi.org/10.1016/j.reu.2015.04.001

18. Elers Y, Pilar M. Relación enfermera-paciente una perspectiva desde las teorías de las relaciones interpersonales. Revista Cubana de Enfermería. 2016; 32(4). [citado Oct 2019]. URL: http://scielo.sld.cu/ pdf/enf/v32n4/enf19416.pdf 
19. Montoya V, Pérez V. Valoración cuantitativa para la reincorporación ocupacional. Salud Uninorte. Revista Científica Salud Uninorte. 2016; 32(2): 319336. [citado Oct 2019]. Disponible en: http://dx.doi. org/10.14482/sun.32.2.8836

20. Iannicelli AM, Matteo P, Vito D, Pellecchia E, Dodaro C, Giallauria F, Vigrito C. Nursing Intervention Classification, Nursing Outcomes Classification and NANDA-NIC-NOC linkage in cardiac rehabilitation. Monaldi Archives for Chest Disease. 2019; 89. [citado May 2020]. Disponible en: https://doi.org/10.4081/ monaldi.2019.1060

21. Herramienta online para la consulta y diseño de Planes de Cuidados de Enfermería. [Internet]. NNNConsult. Elsevier; 2015 [citado Oct 2019]. URL: http://www. nnnconsult.com/

22. Campos de Carvalho E, Appoloni Eduardo AH, Romanzini A, Prado Simao T, Garbuio DC, Heather Herdman T. Correspondence Between NANDA International Nursing Diagnoses and Outcomes as Proposed by the Nursing Outcomes Classification. Journal of Nursing Knowledge. 2016; 29(1), 6678. [citado May 2020]. Disponible en: https://doi. org/10.1111/2047-3095.12135

23. Lee E, Park H. Estimated Time to Complete Direct Nursing Interventions Using the Nursing Interventions Classification (NIC) at Eight Hospitals in South Korea. International Journal of Nursing Knowledge. 2016; 29(2), 104-111. [citado May 2020]. Disponible en: https://doi.org/10.1111/2047-3095.12166

24. Gonzáles Espangler L, Durán Vásquez WE, Ramírez Quevedo Y, Leyet Martínez MR, Cabrera Sánchez VC. Relación de la postura corporal con las maloclusiones en adolescentes de un área de salud. MEDISAN. 2016; 20(12): 6001-6008. [citado May 2020]. URL: https:// www.redalyc.org/articulo.oa? id=3684/368448864001

25. Heon S, Sung Park D. The Effects of Trunk Exercise on Mobility, Balance and Trunk Control of Stroke Patients. J Korean Soc Phys Med. 2017; 12(1): 2533. [citado May 2020]. Disponible en: http://dx.doi. org/10.13066/kspm.2017.12.1.25

26. Trochimczuk R. Huscio T, Grymek S, Szalewska D. Rehabilitation device supporting active and passive upper limb exercises. Current science. 2018; 115(5). [citado May 2020]. Disponible en: https://doi. org/10.18520/cs/v115/i5/868-873

27. Yvonne Chan HB, Ying Pua P, How How C. Physical therapy in the management of frozen shoulder. Singapore Med J. 2017; 58(12): 685-689. [citado May 2020]. URL: https://www.ncbi.nlm.nih.gov/pmc/ articles/PMC5917053/pdf/SMJ-58-685.pdf

28. Takahashi T, Takeshima N, Rogers NL, Rogers ME, Monirul Islam M. Passive and active exercises are similarly effective in elderly nursing home residents.
J Phys Ther Sci. 2015; 27: 2895-2900. [citado May 2020]. Disponible en: https://doi.org/10.1589/ jpts.27.2895

29. Capelari TV, Borin JS, Saccani R, Zardo F, Cechetti F. Evaluation of muscle strength in medullar injury: a literature review. Coluna/Columna. 2017; 16(4): 3239. [citado May 2020]. Disponible en: http://dx.doi. org/10.1590/s1808-185120171604179802 